\title{
On the Statistical Distribution of Packets Service Time in Cellular Access Networks
}

\author{
Ali Y. Al-Zahrani \\ Department of Electrical and Electronic Engineering \\ University of Jeddah \\ Jeddah, Saudi Arabia \\ ayalzahrani1@uj.edu.sa
}

\begin{abstract}
A cellular communication system is divided into two main parts, core network, and radio access network. This research is concerned with the radio access network part which consists of multiple-cells, each served by a central located base station. Furthermore, the users in each cell are considered to be uniformly distributed inside the cell. In the downlink context, the users' packets usually arrive at the base station via fiber optic and then are relayed to the users via radio waves of certain frequency/ies. The speeds of delivering users' packets vary, depending on the users' location. In this paper, the actual distribution of the service time over different users whose locations are uniformly distributed in a cell served by one base station is analytically found. Simulation results are presented to validate the derived model.
\end{abstract}

Keywords-wireless network; cumulative distribution function; probability density function; packet service time; resource allocations

\section{INTRODUCTION}

Deploying a wireless system can be quite costly. Therefore, vendors and operators do extensive simulations before deploying any system to make sure that it is going to work properly as anticipated, and hence the investment will pay off. A key issue for drawing useful insights out of these numerical experiments (i.e. simulations) is the accuracy of the models that represent different parts of the system. In the cellular radio access network (RAN) where each cell is served by one central base station (BS), users are usually distributed within the cell according to a uniform distribution. In addition, the users' packets arrive to the base station via fiber optic channels, and are relayed to the users via wireless radio channels [1,2]. From queuing theory perspective, when the packets arrive at a service facility according to Poison distribution (i.e. the packets interarrival time is an exponential random variable), and being serviced in a time that is exponentially distributed, then the resulted queue is denoted by $\mathrm{M} / \mathrm{M} / 1$. $\mathrm{M}$ stands for "Memoryless" which is a property of the exponential distribution, and 1 indicates the existence of only one server in the facility. This model is the simplest among different queue models [3]. BS may be looked at as the service facility which serves the incoming packets by relaying them to their respective users. This paper aims to show that the resulting queue in the BS cannot be $\mathrm{M} / \mathrm{M} / 1$ as some researchers assume
$[4,5]$. Since the backhaul of the BS is connected to a single reliable fiber optic channel, the inter-arrival time of the packets to the BS may be modeled as an exponential random variable [6]. On the other hand, the time required to send packets is a random variable, but cannot be modeled as exponentially distributed because these packets belong to many users with different channels to the BS. Hence, the packets are delivered with completely different data rates. The main contributions of this paper are:

- The statistical distribution of the service time required to deliver packets from the BS to the users is analytically derived.

- A function for generating service time samples, which is a very useful tool for simulation purposes, is provided.

- The statistical distribution of the service rate under the described system setup is also derived.

- The impact of the derived service time distribution is highlighted from the queuing theory perspective.

\section{SYSTEM DESCRIPTION}

In a typical cell of a radius $r$, as shown in Figure 1, users are distributed within the cell according to a uniform distribution. Hence, users generally experience different pathloss due to their different distances $d$ from the BS. Furthermore, since the BS backhaul is a reliable fiber optic link, the users' packets arrive to the cell BS according to the Poisson distribution with an average rate of $\lambda$ packets per second. For simplicity, we assume that the considered BS has one frequency channel $f_{c}$ which is allocated/shared among users' packets over time domain according to the First-in-Firstout policy (FIFO). Considering the BS as the router which routes the arrived packets to their respective users, the service time required to send each packet depends on the channel gain from the BS to the packet's destination. The channel gain $g$ from a user distant from the BS by $d(\mathrm{~m})$ is $g=C\left(\frac{d_{o}}{d}\right)^{\alpha}$, where $C$ is a constant $\left(\mathrm{C}=\mathrm{A}_{\mathrm{G}}\left(\frac{\lambda_{\mathrm{s}}}{4 \pi \mathrm{d}_{\mathrm{o}}}\right)^{2}\right.$, with $A_{G}$ being the overall antenna gain, and $\lambda_{s}$ the signal wavelength), $d_{o}$ is the reference distance, and $\alpha$ is the pathloss exponent. Finally, we assume the size of each packet is $L$ bits. 


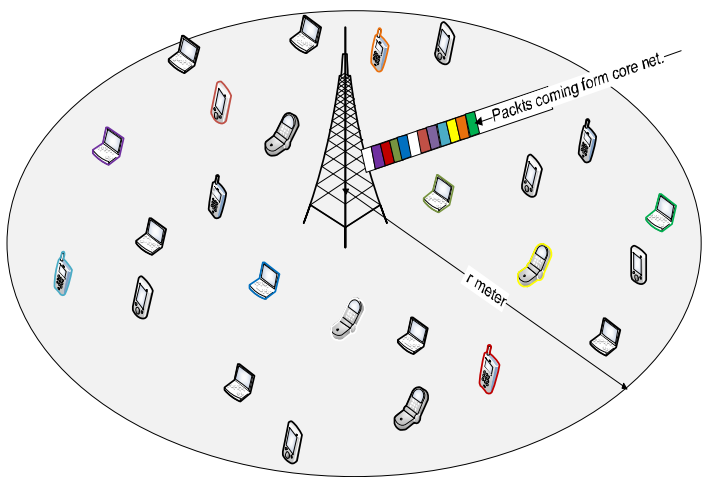

Fig. 1. A radio access network

III. SYSTEM ANALYSIS

Since the mobile users are uniformly distributed within the cell, the distance $D$ from the base station to any randomly selected user is a random variable (RV) whose cumulative distribution function (CDF) can be found as follows. Considering only the users with the cell, the probability that a typical user is within an area of radius $d$ such that $d_{o}<d<r$ is given by:

$$
\begin{gathered}
\frac{\text { internal disc area }}{\text { total area of the effective cell }}=\frac{\int_{d_{o}}^{d} \int_{0}^{2 \pi} x d \theta d x}{\pi\left(r^{2}-d_{o}^{2}\right)} \\
=\frac{\int_{d_{o}}^{d} 2 \pi x d x}{\pi\left(r^{2}-d_{o}^{2}\right)}
\end{gathered}
$$

Solving the above equation yields the CDF of the distance from the BS the a randomly selete user:

$$
\begin{gathered}
F_{D}(d)=P[D \leq d] \\
F_{D}(d)=\left\{\begin{array}{ccc}
0 & \text { for } & d \leq d_{o} \\
\frac{d^{2}-d_{o}^{2}}{r^{2}-d_{o}^{2}} & \text { for } & d_{o} \leq d \leq r, \\
1 & \text { for } & d \geq r
\end{array}\right.
\end{gathered}
$$

where $d$ is any typical distance and $r$ is the radius of the cell. Thus, the average channel gain $G$ from the base station to any randomly selected user is a random variable and can be defined as a function of the distance $D$ :

$$
G=C\left(\frac{d_{o}}{D}\right)^{\alpha}
$$

Therefore, the CDF of the channel gain can be found as [9]:

$$
\begin{gathered}
F_{G}(g)=P[G \leq g]=P\left[C\left(\frac{d_{o}}{D}\right)^{\alpha} \leq g\right] \\
=P\left[D g^{1 / \alpha} \geq C^{1 / \alpha} d_{o}\right]=P\left[D \geq \frac{d_{o} C^{1 / \alpha}}{g^{1 / \alpha}}\right] \\
=1-P\left[D \leq d_{o}\left(\frac{C}{g}\right)^{1 / \alpha}\right] \\
=1-F_{D}\left(d_{o}\left(\frac{C}{g}\right)^{\frac{1}{\alpha}}\right)
\end{gathered}
$$

Substituting (1) into the last equation yields:

$$
\begin{aligned}
& F_{G}(g)= \\
& 1-\left\{\begin{array}{ccc}
0 & \text { for } & d_{o}\left(\frac{C}{g}\right)^{1 / \alpha} \leq d_{o} \\
\frac{\left(d_{o}\left(\frac{c}{g}\right)^{1 / \alpha}\right)^{2}-d_{o}^{2}}{r^{2}-d_{o}^{2}} & \text { for } & d_{o} \leq d_{o}\left(\frac{C}{g}\right)^{1 / \alpha} \leq r \\
1 & \text { for } & d_{o}\left(\frac{C}{g}\right)^{1 / \alpha} \geq r
\end{array}\right.
\end{aligned}
$$

After rearranging (2), we obtain the CDF of the channel gain of a randomly selected user as follows:

$$
=\left\{\begin{array}{clc} 
& F_{G}(g)= \\
0 & \text { for } & g \leq C\left(\frac{d_{o}}{r}\right)^{\alpha} \\
1-\frac{d_{o}^{2}\left(C^{2 / \alpha}-g^{2 / \alpha}\right)}{g^{2 / \alpha}\left(r^{2}-d_{o}^{2}\right)} & \text { for } & C\left(\frac{d_{o}}{r}\right)^{\alpha} \leq g \leq C \\
1 & \text { for } & g \geq C
\end{array}\right.
$$

Assuming the system applies capacity achieving code, the throughput of a given user, whose channel gain is $g$, will be $R=B \log _{2}\left(1+\frac{p g}{B N_{o}}\right)$, where $p$ is the a constant transmit power, $B$ is the channel bandwidth, and $N_{o}$ is the noise singlesided power spectral density. Furthermore, the service time required to transmit one packet of $L$ bits over one frequency band, form the BS to a randomly selected user is a random variable $T=\frac{L}{R}$. The CDF of the service time $F_{T}(t)$ is derived as follows:

$$
\begin{gathered}
F_{T}(t)=P[T \leq t]=P\left[\frac{L}{B \log _{2}\left(1+\frac{p G}{B N_{o}}\right)} \leq t\right] \\
=P\left[\frac{L}{B t} \leq \log _{2}\left(1+\frac{p G}{B N_{o}}\right)\right] \\
=P\left[G \geq\left(2^{\frac{L}{B t}}-1\right) \frac{B N_{o}}{p}\right] \\
=1-P\left[G \leq\left(2^{\frac{L}{B t}}-1\right) \frac{B N_{o}}{p}\right] \\
\Rightarrow F_{T}(t)=1-F_{G}\left(\left(2^{\frac{L}{B t}}-1\right) \frac{B N_{o}}{p}\right)
\end{gathered}
$$

Substituting (3) into (4) yields the CDF of the service time over one frequency channel as shown below:

$$
\begin{aligned}
& \mathrm{F}_{\mathrm{T}}(\mathrm{t})= \\
& \left\{\begin{array}{ccc}
0 & \text { for } & \mathrm{t} \leq \frac{\mathrm{L}}{\mathrm{Blog}_{2}\left(1+\frac{\mathrm{PC}}{\mathrm{BN}}\right)} \\
\mathrm{k} \frac{\mathrm{C}^{\frac{2}{\alpha}}-\mathrm{h}(\mathrm{t})}{\mathrm{h}(\mathrm{t})} & \text { for } & \frac{\mathrm{L}}{\mathrm{Blog}_{2}\left(1+\frac{\mathrm{PC}}{\mathrm{BN}}\right)} \leq \mathrm{t} \leq \frac{\mathrm{L}}{\operatorname{Blog}_{2}\left(1+\frac{\mathrm{PC}\left(\frac{\mathrm{d}_{\mathrm{o}}}{\mathrm{r}}\right)^{\alpha}}{\mathrm{BN} \mathrm{N}_{\mathrm{O}}}\right)}
\end{array}\right. \\
& 1 \text { for } \mathrm{t} \geq \frac{\mathrm{L}}{\operatorname{Blog}_{2}\left(1+\frac{\mathrm{PC}\left(\frac{\mathrm{d}_{\mathrm{o}}}{\mathrm{r}}\right)^{\alpha}}{\mathrm{BNo}}\right)} \\
& \text { where } k=\frac{d_{o}^{2}}{r^{2}-d_{o}^{2}} \text {, and } h(t)=\left(2^{\frac{L}{B t}}-1\right)^{\frac{2}{\alpha}}\left(\frac{B N}{P}\right)^{\frac{2}{\alpha}} \text {. }
\end{aligned}
$$




\section{A. Generating Service Time Samples}

For simulation purposes, it is sometimes required to generate samples of service time. The inverse-transform technique can be used for generating samples of service time. Since $F_{T}(t) \in[0,1]$, and is a monotone non-decreasing function, $N$ samples of the service time can be generated by: $\left\{T_{i}=F_{T}^{-1}\left(U_{i}\right)\right\}_{i=1}^{N}$, where $F_{T}^{-1}$ is the inverse of the CDF in (5), and $\left\{U_{i}\right\}_{i=1}^{N}$ are randomly generated samples that follow the uniform distribution over the interval $[0,1]$. Below, we explicitly find $F_{T}^{-1}$ :

$$
\begin{gathered}
U=k \frac{C^{2 / \alpha}-h(T)}{h(T)} \\
\frac{U h(T)}{k}=C^{2 / \alpha}-h(T) \\
h(T)\left[\frac{U}{k}+1\right]=C^{2 / \alpha} \\
h(T)=\frac{k C^{2 / \alpha}}{U+k} \\
\left(2^{\frac{L}{B T}}-1\right)^{2 / \alpha}\left(\frac{B N}{P}\right)^{2 / \alpha}=\frac{k C^{2 / \alpha}}{U+k}
\end{gathered}
$$

Solving for $T$ yields:

$$
T=F_{T}^{-1}(U)=\frac{L}{B \log _{2}\left(1+\left(\frac{k}{U+k}\right)^{\alpha / 2} \frac{C P}{B N_{o}}\right)}
$$

Thus, (6) is a direct simulation tool for generating samples of the service time required to send a packet of $L$ bits from the BS to a randomly selected user.

\section{B. Service Time Probability Density Function (PDF)}

From (5), the PDF of the service time can be found:

$$
\begin{gathered}
f_{T}(t)=\frac{d\left(F_{T}(t)\right)}{d t} \\
=\left\{\begin{array}{ccc}
k \frac{-h^{\prime}(t) C^{\frac{2}{\alpha}}}{h^{2}(t)} & \text { for } & \frac{L}{B \log _{2}\left(1+\frac{P C}{B N_{o}}\right)} \leq t \leq \frac{L}{B \log _{2}\left(1+\frac{P C\left(\frac{d_{o}}{r}\right)^{\alpha}}{B N_{o}}\right)} \\
0 & \text { otherwise }
\end{array}\right.
\end{gathered}
$$

where:

$$
h^{\prime}(t)=\frac{d(h(t))}{d t}=-\left(\frac{B N_{o}}{P}\right)^{2 / \alpha} \frac{2 L \ln (2)}{B \alpha} \frac{(2)^{\frac{L}{B t}}\left(2^{\frac{L}{B t}}-1\right)^{\frac{2-\alpha}{\alpha}}}{t^{2}}
$$

After some mathematical manipulations, the PDF of the service time can be rewritten as shown bleow:

$$
\begin{gathered}
f_{T}(t)= \\
\left\{\begin{array}{c}
\psi \frac{1}{t^{2}}\left(2^{\frac{2 \theta}{t}}-2^{-\frac{\alpha \theta}{t}}\right)^{-\frac{2+\alpha}{\alpha}} \text { for } \frac{L}{B \log _{2}\left(1+\frac{P C}{B N_{o}}\right)} \leq t \leq \frac{L}{B \log _{2}\left(1+\frac{P C\left(\frac{d_{o}}{r}\right)^{\alpha}}{B N_{o}}\right)}(9) \\
0 \text { otherwise }
\end{array}\right.
\end{gathered}
$$

where $\psi=k C^{2 / \alpha}\left(\frac{P}{B N_{o}}\right)^{2 / \alpha} \frac{2 L \ln (2)}{B \alpha}$, and $\theta=\frac{L}{B(2+\alpha)}$. Note that both $\psi$ and $\theta$ are constant.
Let the limits of the service time wheren the PDF is non zero be: $t_{o}=\frac{L}{B \log _{2}\left(1+\frac{P C}{B N_{o}}\right)}$, and $t_{e}=\frac{L}{B \log _{2}\left(1+\frac{P C\left(\frac{d_{o}}{r}\right)^{\alpha}}{B N_{o}}\right)}$. Then, the first two moments of the service time are given by [9]:

$$
\begin{aligned}
& E[T]=\psi \int_{t_{o}}^{t_{e}} \frac{1}{t}\left(2^{\frac{2 \theta}{t}}-2^{-\frac{\alpha \theta}{t}}\right)^{-\frac{2+\alpha}{\alpha}} d t \\
& E\left[T^{2}\right]=\psi \int_{t_{o}}^{t_{e}}\left(2^{\frac{2 \theta}{t}}-2^{-\frac{\alpha \theta}{t}}\right)^{-\frac{2+\alpha}{\alpha}} d t
\end{aligned}
$$

\section{Density Function of the Service Rate}

In certain occasions, we are sometimes interested in service rate rather than service time. The service rate $(S)$ is a discrete random variable which represents the number of transmitted packets in one second. In this subsection, we introduce the $\mathrm{CDF}$ and PDF of service rate. Clearly, the relation between service time and service rate is $T=1 / S$. Therefore, The CDF of the service rate can be found as:

$$
\begin{aligned}
& F_{S}(s)=P[S \leq s]=P\left[\frac{1}{T} \leq s\right] \\
& =1-P\left[T \leq \frac{1}{s}\right]=1-F_{T}\left(\frac{1}{S}\right)
\end{aligned}
$$

By substituting (5) in the above equation, we obtain the $\mathrm{CDF}$ of the service rate over one frequency channel as follows:

$$
F_{S}(s)=\left\{\begin{array}{ccc}
0 & \text { for } & s \leq \frac{B \log _{2}\left(\begin{array}{c}
P C\left(\frac{d_{o}}{r}\right)^{\alpha} \\
B N_{o}
\end{array}\right)}{L} \\
(k+1) \frac{u(s)-\frac{k}{k+1} C^{2 / \alpha}}{u(s)} & \text { for } & \frac{B \log _{2}\left(1+\frac{P C\left(\frac{d_{o}}{r}\right)^{\alpha}}{B N_{o}}\right)}{L} \leq s \leq \frac{B \log _{2}\left(1+\frac{P C}{B N_{o}}\right)}{L} \\
1 & \text { for } & s \geq \frac{B \log _{2}\left(1+\frac{P C}{B N_{o}}\right)}{L}
\end{array}\right.
$$

where $u(s)=h\left(\frac{1}{s}\right)$. Furthermore, the PDF of the service rate is given by:

$$
\begin{aligned}
& f_{S}(s)= \\
& =\left\{\begin{array}{c}
\psi\left(2^{2 \theta s}-2^{-\alpha \theta s}\right)^{-\frac{2+\alpha}{\alpha}} \text { for } \frac{B \log _{2}\left(1+\frac{P C\left(\frac{d_{o}}{r}\right)^{\alpha}}{B N_{o}}\right)}{L} \leq s \leq \frac{B \log _{2}\left(1+\frac{P C}{B N_{o}}\right)}{L} \\
0 \text { otherwise. }
\end{array}\right.
\end{aligned}
$$

The average service rate can be found by [9]:

$$
\bar{S}=\int_{\frac{1}{t_{e}}}^{\frac{1}{t_{o}}} s \psi\left(2^{2 \theta s}-2^{-\alpha \theta s}\right)^{-\frac{2+\alpha}{\alpha}} d s
$$

\section{The Impact of the Derived Service Rate}

The BS usually has a number of orthogonal frequency channels through which the transmitters within the BS can transmit the packets to the respective users. When all radio frequencies are assigned to only one transmitter which transmits all packets in a FIFO fashion, then, according to the queuing theory [3], this kind of queue is called M/G/1 queue. $\mathrm{M}$ indicates a Markovian arrival model, in which the packet 
inter-arrival time follows an exponential distribution while $G$ indicates a general service model where the packet service time follows an arbitrary distribution as the one shown in (5), and " 1 " indicates that only one transmitter is in service. When $\lambda<\bar{S}$, the utilization of the BS transmitter is $\rho=\frac{\lambda}{\bar{S}}$, which quantifies the proportion of time the BS transmitter is busy in the long run. Moreover, the system will be stable since $\rho<1$, and the steady-state parameters of this $\mathrm{M} / \mathrm{G} / 1$ queue will be as follows [12]:

- Packet average delay:

$$
\tau=\frac{1}{\bar{S}}+\frac{\lambda\left(1 / \bar{S}^{2}+\sigma_{T}^{2}\right)}{2(1-\rho)}
$$

where $\sigma_{T}^{2}$ is the variance of the packet service time T.

- Average number of packets in the BS:

$$
N=\rho+\frac{\rho^{2}\left(1+\sigma^{2} \bar{S}^{2}\right)}{2(1-\rho)}
$$

- Probability of zero packets at the BS:

$$
P_{0}=1-\rho
$$

If, however, the service model was not general $(\mathrm{G})$, but rather Markovian (M) (as it is usually erroneously assumed), then the steady-state parameters would have been [12]:

- Packet average delay:

$$
\tau=\frac{1}{\bar{S}-\lambda}
$$

- Average number of packets in the BS:

$$
N=\frac{\rho}{1-\rho}
$$

- Probability of $n$-packets at the BS:

$$
P_{n}=\rho^{n}(1-\rho)
$$

These quantities, which are based on loose assumptions, are misleading as they are completetly different than the actual pararmeters shown in (14) and (15). Note that the true packet average delay in (14) is directly proportional to the variance of the service time which indicates that the packet delay increases as the variation in service time increases. Thus, as the users become more scattered in the cell, then their service time, which depenends on the distance from the BS, will vary more. One way to reduce $\sigma_{T}^{2}$ is by dividing the set of all radio frequencies into $N$ subsets assingend to $N$ transmitters for serving $N$ packets simultanously. In addition, each transmitter should serve packets related to users at approximately the same distance from the BS (i.e. a group of user apart from the BS by $d \pm \epsilon)$.

\section{NUMERICAL EXPERIMENT}

In this section, the results of computer simulation are shown and discussed. The simulation is conducted in accordance with the system setup explained above. The simulation parameters are shown in Table I. The simulated network architecture is shown in Figure 2, where the BS (red star) is located at the cell center and all users (black dots) are uniformly distributed in the cell. MATLAB version R2013a was used. The simulation started by randomly choosing the location of each user (i.e. its distance from the base station and its angle with respect to the central point of the cell). Then, the channel of each user was estimated via calculating the signal path loss and small scale fading. By knowing the user channel, data rate can be estimated, and so the required time for delivering each packet. The objective was to measure the required time for delivering each packet, and then draw the CDF of these samples using the MATLAB command cdfplot, and finally comparing the result with analytical CDF found in (5).

TABLE I. SIMULATION PARAMETERS

\begin{tabular}{|c|c|c|}
\hline Parameters & Value & Unit \\
\hline System frequency & 2.5 & $\mathrm{GHz}$ \\
\hline Channel bandwidth & 30 & $\mathrm{KHz}$ \\
\hline Pathloss exponent $(\alpha)$ & 3.52 & - \\
\hline Reference distance $\left(d_{o}\right)$ & 20 & $\mathrm{~m}$ \\
\hline Cell radius & 500 & $\mathrm{~m}$ \\
\hline Transmit power $(p)$ & 0 & $\mathrm{dBm}$ \\
\hline Noise power spectral density & -174 & $\mathrm{dBm} / \mathrm{Hz}$ \\
\hline Overall antenna gain & 0 & $\mathrm{~dB}$ \\
\hline Packet size $(L)$ & 1500 & bits \\
\hline
\end{tabular}

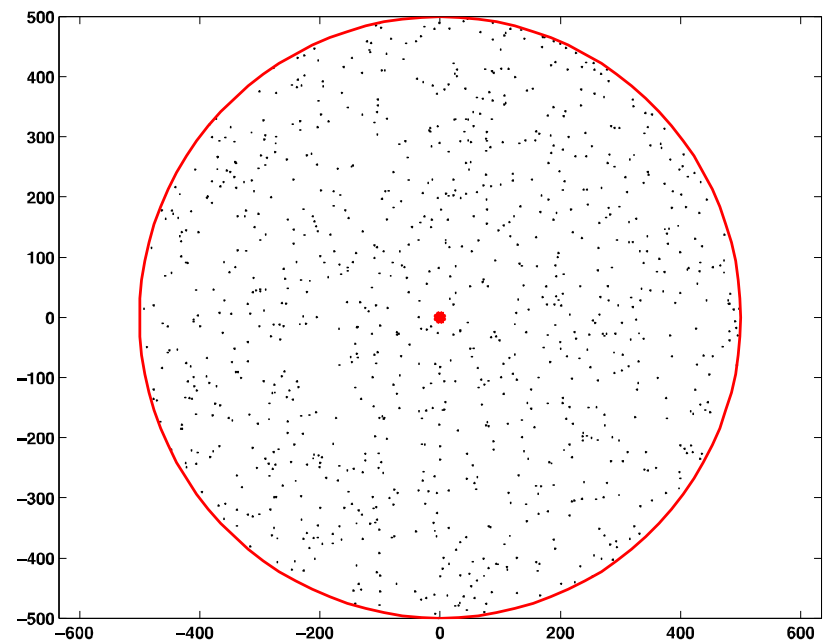

Fig. 2. A simulated cell with $500 \mathrm{~m}$ radius

Figure 3 shows different graphs for the analytical and the simulated CDFs of the time required to relay the packets to the users. The analytical CDF is a simple graph of $F_{T}(t)$ described by (5) while the simulated CDF is based on the data drawn from the simulation. As depicted in the Figure, the simulated CDF fits the analytical CDF. In addition, as the number of users increases, the precision of the fitness increases further, simply because the analytical CDF is based on the limit (i.e. the number of users tends to infinity). This result clearly proves the accuracy of the statistical model of the packet service time proposed in (5). Furthermore, Figure 4 shows a comparison between the analytical PDF portrayed by (9) and the histogram of the service time results drawn from the simulation. The analytical PDF clearly matches the result of the simulation. This shows that the derived statistical model is realistic and closely matches the data-based results. 

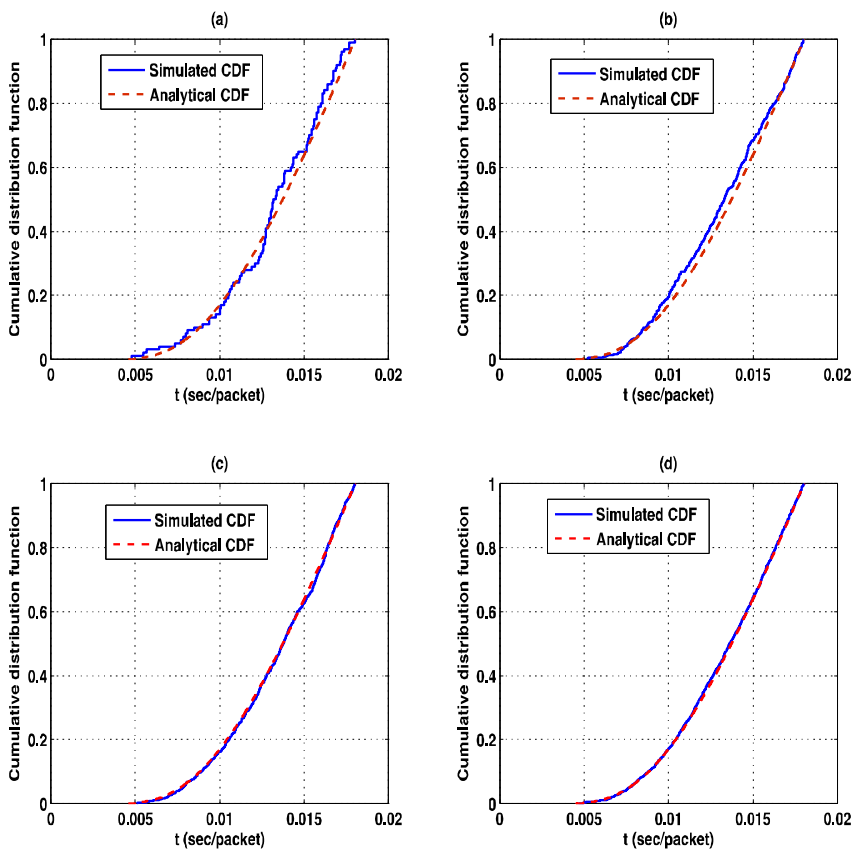

Fig. 3. Comparisons between analytical CDF and simulated CDF: (a) 100 users/cell, (b) $500 \mathrm{users} /$ cell, (c) $1000 \mathrm{users} /$ cell, (d) $2000 \mathrm{users} /$ cell

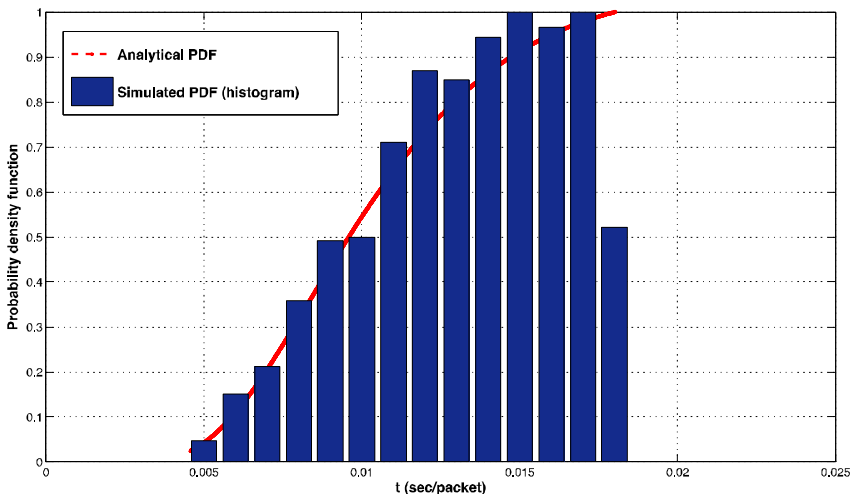

Fig. 4. Comparison between analytical PDF and the histogram of the packet service time

\section{CONCLUSION}

In this paper, the correct statistical model of the time taken by the BS to relay a user's packet was derived. We showed that it is completely different than exponential distribution as it might be assumed. Furthermore, we showed how this can be handy and beneficial in numerical experiments involving the cellular system. Finally, we derived a direct closed form of the $\mathrm{CDF}$ and PDF of the service rate measured in packets per seconds. These results may be beneficial in developing new methods for radio resource management aiming at reducing the waiting time at the BS.

\section{REFERENCES}

[1] T. S. Rappaport, Wireless communication: principles and practice, Prentice Hall, second edition, 2002

[2] A. Goldsmith, Wireless Communication, Cambridge University Press, first edition, 2005
[3] D. P. Bertsekas, R. G. Gallager, Data networks, Prentice-Hall, 1992

[4] K. Son, H. Kim, Y. Yi, B. Krishnamachari, "Base station operation and user association mechanisms for energy-delay tradeoffs in green cellular networks", IEEE Journal on Selected Areas in Communications, Vol. 29, pp. 1525-1536, 2011

[5] H. Kim, G. de Veciana, X. Yang, M. Venkatachalam, “ $\alpha$-optimal user association and cell load balancingin wireless networks", IEEE/ACM Transactions on Networking, Vol. 20, No. 1, pp. 177-190, 2002

[6] D. Niyato, E. Hossain, "Adaptive fair subcarrier/rate allocation in multi rate ofdma networks: radio link level queuing performance analysis", IEEE Transactions on Vehicular Technology, Vol. 55, No. 6, pp. 1897 1907, 2006

[7] A. Y. Al-Zahrani, "Modelling and qos-achieving solution in full-duplex cellular systems", International Journal of Computer Networks \& Communications, Vol. 10, pp. 117-135, 2018

[8] S. Buyukcorak, G. K. Kurt, O. Cengaver, "A probabilistic framework for estimating call holding time distributions", IEEE Transactions on Vehicular Technology, Vol. 63, No. 2, pp. 811-821, 2014

[9] A. Leon-Garcia, Probability and random processes for electrical engineering, Addison-Wesley, 1994

[10] M. Azhar, A. Shabbir, "5G networks: challenges and techniques for energy efficiency", Engineering, Technology \& Applied Science Research, Vol. 8, No. 2, pp. 2864-2868, 2018

[11] L. Scalia, K. K. T. Biermann, C. Choi, W. Kellerer, "Power-efficient mobile backhaul design for CoMP support in future wireless access systems", 2011 IEEE Conference on Computer Communications Workshops, Shanghai, China, April 10-15, 2011

[12] J. Banks, J. S. Carson II, B. L. Nelson, D. M. Nicol, Discrete-event system simulation, Prentice Hall, fourth edition, 2005

\section{AUTHORS PROFILE}

Ali Y. Al-Zahrani received his BSc in Electrical Engineering (with honors) from King Fahad University of Petroleum and Minerals (KFUPM), Dhahran, Saudi Arabia, in 2002 and his MSc and PhD degrees in Electrical and Computer Engineering from Carleton University, Ottawa, ON, Canada, in 2010 and 2015 respectively. He is currently an Assistant Professor at the Department of Electrical and Computer Engineering, University of Jeddah, Saudi Arabia. From 2002 to 2007 he worked as an electrical engineer at the Saudi Basic Industries Corporation (SABIC). His research interests include radio resource allocation and interference management in wireless communication systems, DSP and massive MIMO. 